\title{
BMJ Open Lipid-lowering drugs and risk of new- onset diabetes: a cohort study using Japanese healthcare data linked to clinical data for health screening
}

\author{
Nobuhiro Ooba, ${ }^{1}$ Soko Setoguchi, ${ }^{2}$ Tsugumichi Sato, ${ }^{3}$ Kiyoshi Kubota ${ }^{4}$
}

To cite: Ooba N, Setoguchi S, Sato T, et al. Lipid-lowering drugs and risk of new-onset diabetes: a cohort study using Japanese healthcare data linked to clinical data for health screening. BMJ Open 2017;7:e015935. doi:10.1136/ bmjopen-2017-015935

- Prepublication history for this paper is available online. To view these files please visit the journal online (http://dx.doi. org/10.1136/bmjopen-2017015935).

Received 11 January 2017 Revised 13 May 2017 Accepted 24 May 2017

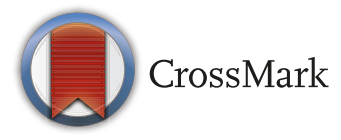

${ }^{1}$ Department of Clinical Pharmacy, Nihon University School of Pharmacy, Funabashi, Chiba, Japan

${ }^{2}$ Institute for Health, Health Care Policy and Aging Research, Rutgers University and Rutgers Robert Wood Johnson Medical School, New Brunswick, New Jersey, USA

${ }^{3}$ Department of Pharmacy, Tokyo University of Science, Yamazaki, Noda, Chiba, Japan

${ }^{4}$ NPO Drug Safety Research Unit Japan, Tokyo, Japan

Correspondence to Dr Kiyoshi Kubota; kubotapetky@umin.net

\section{ABSTRACT}

Objective To investigate whether lipid-lowering drugs are associated with new-onset diabetes after adjusting for baseline clinical risk factors for diabetes.

Design A retrospective cohort study.

Setting Japanese employees of large corporations and their dependents using health insurance claims data linked to clinical and laboratory data for annual health screenings.

Participants All persons aged 20 to 74 years with dyslipidaemia between 1 January 2005 and 31 March 2011. We defined the index date as the first date when the person met the criteria for dyslipidaemia. Persons were excluded if they had lipid-lowering drugs, or had a diagnosis, a treatment or a laboratory test result (haemoglobin A1c $\geq 6.5 \%$ or fasting blood glucose $\geq 126 \mathrm{mg} / \mathrm{dL}$ ) indicating diabetes during the 6-month period before the index date.

Main outcome measures New-onset diabetes.

Results We identified 68620 persons with dyslipidaemia. During the mean follow-up period of 1.96 years, 3674 persons started treatment with a lipid-lowering drug: 979 with a low potency statin, 2208 with a high potency statin and 487 with a fibrate. Of 3674 new users of a lipid-lowering drug, 3621 had a period of non-use of any lipid-lowering drugs before starting a lipid-lowering drug. Among statin users, the incidence rate of new-onset diabetes was 124.6 per 1000 person-years compared with 22.6 per 1000 person-years in non-users. After adjusting for confounding factors including clinical data in health screening using Cox proportional hazards models, the HR was 1.91 (95\% Cl 1.38 to 2.64 ) for low potency statins and 2.61 (2.11 to 3.23) for high potency statins.

Conclusion The use of statins was associated with a 1.9fold to 2.6-fold increase in the risk of new-onset diabetes in a Japanese population of working age, despite adjusting for clinical risk factors for diabetes.

\section{INTRODUCTION}

Hyperlipidaemia is an established risk factor for cardiovascular diseases. In Japan, the estimated number of patients with hyperlipidaemia was approximately 1.9 million at 2011. ${ }^{1}$ Statins are widely used for primary and secondary prevention of cardiovascular diseases $^{2}$ and have a good safety profile in

\section{Strengths and limitations of this study}

- This was not a randomised controlled trial but a retrospective cohort study using health insurance claims data linked to clinical and laboratory data for annual health screenings and subject to the confounding bias even if the bias was adjusted as much as possible.

- Our study population included those who worked in relatively large corporations and their relatives, aged between 20 and 74 years, which means our findings might not be readily generalised to those working in small corporations or self-employed individuals and old people aged $\geq 75$ years.

- The diagnosis code for diabetes was not validated in our database, and therefore the outcome in sensitivity analyses was defined using an antidiabetic drug with or without the diagnosis code for diabetes.

- Although the information on some of potential confounders such as smoking, alcohol consumption and physical exercise was not available, we could adjust for several important risk factors of diabetes such as $\mathrm{HbA1C}$ and fasting blood glucose essential in diagnosis of diabetes, as well as body mass index, blood pressure, uric acid, low-density lipoprotein cholesterol cholesterol, high-density lipoprotein cholesterol cholesterol, triglyceride and urinary glucose.

- Our study suggested that the use of statins was associated with an increased risk of new-onset diabetes, compared with the period of non-use, despite adjusting for risk factors for diabetes (eg, $\mathrm{HbA1c}$ and body mass index) obtained in the annual health screening.

- We also found that the risk of new-onset diabetes using high potency statins (atorvastatin, rosuvastatin and pitavastatin) was higher than that of low potency statins (fluvastatin, pravastatin and simvastatin).

general, although rare adverse reactions such as liver dysfunction and rhabdomyolysis can occur. ${ }^{3}$ According to recent studies, the use of statins is associated with an increased risk of new-onset diabetes. ${ }^{4-6}$ As with 
hyperlipidaemia, diabetes is an established risk factor for cardiovascular diseases, ${ }^{7}$ and therefore the possible increased risk of new-onset diabetes by the drugs used to treat hyperlipidaemia may attenuate the effect of reducing the risk of cardiovascular diseases.

Meta-analyses of randomised trials report that the use of statins was associated with a modestly increased risk of diabetes (overall OR=1.09, 95\% CI: 1.02 to 1.17 ) and the OR for individual statins ranged from 0.98 to $1.18 .{ }^{4}$ However, randomised trials may not accurately represent actual practice or patients. The results from observational studies are not consistent. A study using data from the women's health initiative found that high potency statins (HR 1.45) and low potency statins (HR 1.48) were similarly associated with the increased risk of diabetes. ${ }^{5}$ On the other hand, in a study using the administrative healthcare database of Ontario, ${ }^{6}$ high potency statins had a slightly higher risk than that of low/moderate potency statins (HR 1.22 vs $0.97 / 1.11$ ). The association between fibrates and new-onset diabetes indicated inconsistent results in a small number of previous studies. ${ }^{89}$

One potential reason for the inconsistency in assessing the magnitude of diabetes risk, arising from previous observational studies, may be due to the unavailability of information on the important confounders, such as cholesterol level, blood pressure, body mass index (BMI), haemoglobin Alc (HbAlc) and fasting blood glucose (FBG). The inability to control for these factors that potentially introduce confounding by indication or severity may have created an association between the use of a statin with new-onset diabetes variably targeting different study populations in previous studies. It is also unclear whether the risk of new-onset diabetes is specific to statins or common to lipid-lowering drugs (LLDs) including fibrates. In addition, there have been few reports on the association between statins and the incidence of diabetes in the Asian population. ${ }^{1011}$ We conducted a cohort study using claims data linked to the health screening data including blood pressure, BMI, HbA1c, FBG and other laboratory test results to assess the association between the use of LLDs and new-onset diabetes, adjusted for each of these factors.

\section{METHODS}

\section{Data sources}

We used three types of data: claims data, data derived from enrolment and data of regular health screening for beneficiaries of dozens of corporate health insurances for workers in private firms and their dependents. Under the Industrial Safety and Health Act, in addition to the Act on Assurance of Medical Care for Elderly People, ${ }^{12}$ all the insurers are required to provide the regular health screening and health guidance to beneficiaries and maintain and use the health screening data for the health guidance. The three types of data (claims, enrolment and health screenings) are linked by the insurers who provide the health guidance to beneficiaries and evaluate the heath guidance outcome. The data for 215307 beneficiaries aged between 20 and 74 years in the study period (1 January 2005 to 31 March 2011) were collected and maintained by Japan Medical Data Centre Co. ${ }^{13}$ Information on healthcare utilisation, such as outpatient visits and hospitalisation, drugs, medical procedures and diagnoses, was available from the claims data. With respect to drug information ${ }^{14}$ within the claims, more than 20000 local codes are used to specify the trade name and dosage form for all approved drugs. Codes for medical procedures include order of laboratory tests such as HbAlc, triglyceride (TG), total cholesterol, low-density lipoprotein (LDL) cholesterol and high-density lipoprotein (HDL) cholesterol. The 10th revision of the International Classification of Diseases was used for diagnoses. The enrolment data contained the dates of enrolment and disenrollment. Annual health screening is mandatory for employees irrespective of whether they have already had cardiovascular and other diseases under the Industrial Safety and Health Act, and optional for their dependents. ${ }^{15}$ The health screening data contained values of BMI, systolic and diastolic blood pressure, total cholesterol, LDL cholesterol, HDL cholesterol, TG, HbA1c, FBG, serum creatinine (SCre), uric acid (UA), urinary glucose and urinary protein.

\section{Study cohort}

We identified 94630 subjects with or without prior cardiovascular diseases who had either total cholesterol $\geq 220 \mathrm{mg} / \mathrm{dL}$, LDL cholesterol $\geq 140 \mathrm{mg} / \mathrm{dL}$, HDL cholesterol $<40 \mathrm{mg} / \mathrm{dL}$ or $\mathrm{TG} \geq 150 \mathrm{mg} / \mathrm{dL}$ measured at the annual health screening period between 1 January 2005 and 31 March 2011 (figure 1). ${ }^{16}$ We defined the index date as the first date when one of these criteria was met and the baseline period as the period when the data on claims and health screening were available, after the date of enrolment but before the index date. Subjects were excluded if they had a baseline period of $<6$ months, had a diagnosis of diabetes, had used an antidiabetic drug or had a test result indicating diabetes $(\mathrm{HbA} 1 \mathrm{c} \geq 6.5 \%$ or FBG $\geq 126 \mathrm{mg} / \mathrm{dL}$ ) within the baseline period. Subjects were also excluded if they had an LLD during the 6 months before the index date, if neither HbAlc nor FBG was available in the baseline period or if two or more LLDs prescribed at one time were filled on the index date.

\section{Exposures and outcomes data}

We categorised person-times of the cohort members into non-use of LLDs and new use of statins or fibrates (bezafibrate and fenofibrate). Statins were further classified into the low (simvastatin, fluvastatin and pravastatin) and high potency statins (atorvastatin, rosuvastatin and pitavastatin), ${ }^{18}$ as the risk of diabetes may be different between low and high potency statins. ${ }^{6} 19$

New-onset diabetes defined as a diagnosis code of diabetes or where an oral antidiabetic drug or insulin was used after the index date. In the sensitivity analyses, we defined new-onset diabetes as the use of an antidiabetic 


\section{0 persons met one of the criteria for dislipidaemia \\ $(\mathrm{TC} \geq 220 \mathrm{mg} / \mathrm{dL}, \mathrm{LDL}-\mathrm{C} \geq 140 \mathrm{mg} / \mathrm{dL}, \mathrm{HDL}-\mathrm{C}<40 \mathrm{mg} / \mathrm{dL}$ or $\mathrm{TG} \geq 150 \mathrm{mg} / \mathrm{dL})$ \\ in health screening between January 1, 2005 and May 31, 2011.}

26010 patients excluded from the study.

11842 patients had the pre-index period less than six months.

9068 patients had diagnosis of diabetes or used an antidiabetic drug,

$\mathrm{HbA} 1 \mathrm{c} \geq 6.5 \%$, or $\mathrm{FBG} \geq 126 \mathrm{mg} / \mathrm{dL}$ in health screening data before the index date.

2667 patients had a prescription of a lipid-lowering drugs within six months before the index date. 2332 patients had neither laboratory data of HbA1c nor FBG before the index date.

101 patients were disenrolled on the index date.

\section{0 patients were selected as study cohort members. \\ Of those with dyslipidaemia, \\ 64,946 had a period of non-use only, \\ 3,621 had a period of non-use prior to the period of lipid-lowering drug, and \\ $53 \mathrm{had}$ a period of lipid-lowering drug only.}

Figure 1 Study flow diagram. FBG, fasting blood glucose; HbA1c, haemoglobin A1c; HDL-C, high-density lipoprotein cholesterol; LDL-C, low-density lipoprotein cholesterol; TC, total cholesterol; TG, triglyceride.

drug after the index date only disregarding the diagnosis code. The end of the observation period for the outcome assessment was defined as either the date of disenrollment or the occurrence of new-onset diabetes or discontinuation of an LLD or switching to or addition of another category of LLD or 31 March 2011, whichever came first. Therefore, the period when the patient used a statin and a fibrate at the same time was excluded from the observation. Continuous use of the LLDs was defined using the grace period of up to 30 days before filling the next prescription. We considered that an LLD was used continuously when an LLD was switched to another LLD under the same category of LLDs (fibrates, high-potency statins and low-potency statins). If two or more LLDs were started on the same date (but after the index date), the observation was censored.

\section{Covariates}

The following covariates were obtained from the claims data and considered for confounding adjustment: age, sex, the use of other prescribed medications (systemic corticosteroids, thiazide diuretics, beta-adrenergic blocking agents, antipsychotics, antihypertensives and nicotinic acid) and a laboratory test order of $\mathrm{HbAlc}, \mathrm{TG}$, LDL cholesterol and HDL cholesterol during the period of 6 months preceding the index date, a prior diagnosis of myocardial infarction, chronic heart failure, cerebrovascular disease, renal, liver and pulmonary diseases, cancer, hypertension and polycystic ovarian syndrome. We selected the covariates that are known risk factors for diabetes (eg, systemic corticosteroid) and other major conditions (eg, cancer). ${ }^{6} 7$ The Charlson Comorbidity Inde ${ }^{20}$ was also estimated and included as a covariate. Clinical covariates obtained from the health screening data included BMI, blood pressure (diastolic blood pressure and systolic blood pressure), FBG, HbAlc, levels of serum TG, cholesterol (LDL cholesterol and HDL cholesterol), UA and urinary protein and urinary glucose, estimated glomerular filtration rate (e-GFR; classified as $\mathrm{e}-\mathrm{GFR} \geq 90,60 \leq \mathrm{e}-\mathrm{GFR}<90,30 \leq \mathrm{e}-\mathrm{GFR}<60$, e-GFR $<30$ ) and presence of metabolic syndrome (MS). We calculated e-GFR using age, sex and measured SCre. ${ }^{21}$ MS was defined according to the criteria by Sicras et al criteria, ${ }^{22}$ where BMI $\left(\geq 28.8 \mathrm{~kg} / \mathrm{m}^{2}\right)$ instead of waist circumference $(>102 \mathrm{~cm}$ in males and $>88 \mathrm{~cm}$ in females) was used to define MS, as data of waist circumference were not available in our data.

These covariates were measured during the baseline period for all subjects and again during the 6 months prior to the first prescription for lipid-lowering medications for those who initiated an LLD during the follow-up period.

\section{Statistical analysis}

We described the baseline characteristics measured during the 6 months prior to the index date for patients with the period of non-use, as well as the baseline characteristics measured on the date of new use of an LLD for new users of LLDs. We calculated crude incidence rates of new-onset diabetes for users and non-users of LLDs. For missing values in the health screening data, we first conducted a complete case analysis, followed by 
a multiple imputation using SAS V. 9.4 PROC MI. While approximately $20 \%$ of values for FBG, SCre, e-GFR and UA were missing, $<8 \%$ were missing for BMI, diastolic or systolic blood pressure, HbA1c, LDL cholesterol, HDL cholesterol, TG, urinary protein and urinary glucose and MS. The Cox proportional hazards regression model was used to estimate unadjusted, age-sex-adjusted and multivariate-adjusted effects of the LLDs on new-onset diabetes. In all analyses, the reference used was the risk of new-onset diabetes in person-time of non-use of any LLDs.

We conducted multiple sensitivity analyses. First, we conducted the analyses including the period after switching of the LLD, although the observation was censored when an LLD was discontinued or switched in the primary analysis. In this sensitivity analysis, we used the latest value of the covariates assuming that they were not affected by the preceding LLD. Second, we conducted analyses using the grace period of up to 15 or 60 days. Third, to evaluate the robustness of our findings for potential outcome misclassification, the outcome was defined as the incident use of antidiabetic drugs only, disregarding whether the diagnosis code of diabetes was recorded. Lastly, to assess the impact of time-varying confounding, we used a marginal structural model (MSM) to analyse the data using the covariate measured after the date of new use of an LLD while the same definition as in the primary analysis was used for the outcome and grace period. ${ }^{23}$ As in the previous study using the database, we used the inverse-probability weight estimated on a monthly basis. ${ }^{24}$ We considered TG as a possible time-varying confounder because the average TG in the patients with fibrates (around $400 \mathrm{mg} / \mathrm{dL}$ ) was much higher than that with statins (around $160 \mathrm{mg}$ / $\mathrm{dL}$ ), and the primary analysis revealed that the risk of new-onset diabetes was associated with TG (in $\mathrm{mg} / \mathrm{dL}$ ) measured on the date of a new use of an LLD (HR 1.0005, 95\% CI: 1.0001 to 1.0008 ). All analyses were performed with SAS V. 9.4 (SAS Institute).

\section{RESULTS}

\section{Study population}

We identified 94630 persons with dyslipidaemia from laboratory test results in health screening. Of those, $68620(73 \%)$ persons met the eligibility criteria and were included in the study cohort (figure 1). Of these with dyslipidaemia, $64946(69 \%)$ had a period of non-use only, 3621 (4\%) had a period of non-use prior to the period of LLD and $53(0.06 \%)$ had a period of LLD only. Thus, $3674(=3621+53)(4 \%)$ had a period of new use of an LLD (statin or fibrate) with or without a period of non-use. Among 3674 initiators of an LLD, 979 (27\%) started with a low potency statin (pravastatin $(n=726)$, fluvastatin $(\mathrm{n}=111)$ and simvastatin $(\mathrm{n}=142)), 2208$ $(60 \%)$ started with a high potency statin (atorvastatin $(\mathrm{n}=704)$, rosuvastatin $(\mathrm{n}=1016)$ and pitavastatin $(\mathrm{n}=488))$ and $487(13 \%)$ started with a fibrate (bezafibrate $(\mathrm{n}=300)$ and fenofibrate $(\mathrm{n}=187))$. Table 1 shows the baseline characteristics. The mean age of patients with a period of non-use was younger than that of new users of an LLD. The proportion of men in new fibrate users was higher than that in new statin users.

\section{LLDs and new-onset diabetes}

The crude incidence rate of new-onset diabetes was 22.6 per 1000 person-years during the period of non-use while that for the use of LLDs was 105.5 for low potency statins, 133.1 for high potency statins and 99.2 per 1000 personyears for fibrates. Compared with non-use, the use of low or high potency statins or fibrates was associated with an increased risk of new-onset diabetes (table 2).

By the complete case analysis using the Cox proportional hazards regression, the risk of new-onset diabetes for high potency statins (HR 3.42, 95\% CI 2.54 to 4.60 ) and low potency statins $(2.04,1.25$ to 3.33$)$ and fibratres $(2.35,1.08$ to 5.15$)$ was found to be higher compared with the risk during the period of non-use. The Cox proportional hazards regression for multiple imputation revealed that the risk of new-onset diabetes for high potency statins (adjusted HR 2.61, 95\% CI 2.11 to 3.23) was 1.4 to 1.6 times higher than that of low potency statins $(1.91,1.38$ to 2.64) and fibrates (1.64, 0.98 to 2.76) (table 2).

In table 3, the unadjusted and adjusted HRs of new-onset diabetes of the individual LLDs, compared with non-use, are shown. All of the individual statins except for simvastatin were associated with an increased risk of new-onset diabetes. The HR was higher than 1 for the individual fibrates, but the CIs were wide and crossed 1.00.

Figure 2 shows the results of the sensitivity analyses. The results were similar to those of the primary analysis when the periods after a total of 164 occasions of switching of an LLD were included as well as when the grace period was set as either 15 or 60 days. When the outcome was defined by the new use of antidiabetic drugs, the HR was greater than 1 but the CI of the HR was wider than that in the primary analysis.

In the analysis using MSM, the results were similar to those in the primary analysis. The HR with the inverse-probability weight was 2.75 (2.23 to 3.39) for high potency statins, 1.67 (1.19 to 2.36) for low potency statins and 1.53 (0.87 to 2.71 ) for fibrates.

\section{DISCUSSION}

In this retrospective cohort study using data of claims, enrolment and health screening, we found that the use of LLDs (statins and fibrates) was associated with an increased risk of new-onset diabetes, compared with non-use. The adjusted HR of new-onset diabetes between two fibrates (bezafibrate and fenofibrate) was similar, while the HR in the high potency statins (atorvastatin, rosuvastatin and pitavastatin) was higher than that in the low potency statins (fluvastatin, pravastatin and simvastatin). The HR for new-onset diabetes was varied from 1.5 to 3.1 in the individual statins. As the 
Table 1 Baseline characteristics of patients with period of use/non-use of lipid-lowering drugs

\section{Patients with period of use of lipid-lowering drugs}

\begin{tabular}{|c|c|c|c|c|}
\hline & $\begin{array}{l}\text { High potency: } \\
\text { atorvastatin, } \\
\text { pitavastatin, } \\
\text { rosuvastatin }\end{array}$ & $\begin{array}{l}\text { Low/moderate } \\
\text { potency: pravastatin, } \\
\text { fluvastatin, } \\
\text { simvastatin }\end{array}$ & $\begin{array}{l}\text { Fibrate: } \\
\text { bezafibrate, } \\
\text { fenofibrate }\end{array}$ & $\begin{array}{l}\text { Patient with } \\
\text { period of non-use }\end{array}$ \\
\hline Number & 2208 & 979 & 487 & 68567 \\
\hline Mean age (SD) & $49.6(9.5)$ & $49.1(9.8)$ & $45.3(9.4)$ & $42.2(9.9)$ \\
\hline Male sex (\%) & 59.6 & 60.6 & 89.7 & 70.2 \\
\hline \multicolumn{5}{|l|}{ Prescribed drugs (\%) } \\
\hline Nicotinic acid & 1.0 & 1.7 & 1.6 & 0.3 \\
\hline Antihypertensive & 26.7 & 25.0 & 24.6 & 5.3 \\
\hline Beta blocker & 3.6 & 3.7 & 3.3 & 0.9 \\
\hline \multicolumn{5}{|l|}{ Comorbidities (\%) } \\
\hline Myocardial infarction & 1.0 & 0.6 & 0.4 & 0.1 \\
\hline Chronic heart failure & 0.7 & 1.0 & 0.6 & 0.1 \\
\hline Cerebrovascular disease & 2.3 & 1.4 & 0.4 & 0.5 \\
\hline Renal disease & 0.7 & 0.9 & 1.2 & 0.2 \\
\hline Liver disease & 3.9 & 4.5 & 8.0 & 0.8 \\
\hline Pulmonary disease & 3.8 & 3.7 & 4.3 & 2.7 \\
\hline Any cancer & 3.7 & 3.1 & 3.0 & 1.3 \\
\hline Polycystic ovarian syndrome & 0 & 0 & 0 & 0.01 \\
\hline$\geq 2$ & 7.2 & 6.0 & 6.8 & 2.2 \\
\hline \multicolumn{5}{|c|}{ Order for laboratory tests in claims (\%) } \\
\hline $\mathrm{HbA1c}$ & 15.2 & 11.1 & 13.6 & 1.3 \\
\hline Triglyceride & 59.2 & 58.9 & 71.5 & 7.3 \\
\hline Total cholesterol & 43.0 & 46.1 & 53.4 & 7.4 \\
\hline LDL cholesterol & 37.3 & 34.7 & 37.4 & 2.7 \\
\hline HDL cholesterol & 45.6 & 45.2 & 52.6 & 4.3 \\
\hline \multicolumn{5}{|l|}{$\begin{array}{l}\text { Laboratory test result in health } \\
\text { screening }\end{array}$} \\
\hline Mean BMI (SD), kg/m² & $24.2(3.7)$ & $23.9(3.5)$ & $25.3(3.5)$ & $23.8(3.6)$ \\
\hline Missing (\%) & 1.9 & 2.5 & 2.7 & 1.5 \\
\hline Mean DBP (SD), mm/Hg & $79(13)$ & $78(12)$ & $81(11)$ & $75(11)$ \\
\hline Missing (\%) & 0 & 0 & 0 & 0.01 \\
\hline Mean SBP (SD), mm/Hg & $128(18)$ & $128(17)$ & $131(16)$ & $124(16)$ \\
\hline Missing (\%) & 0 & 0 & 0 & 0.01 \\
\hline Mean HbA1c (SD), \% & $5.2(0.3)$ & $5.2(0.3)$ & $5.1(0.4)$ & $5.0(0.3)$ \\
\hline Missing (\%) & 3.7 & 3.7 & 2.3 & 3.0 \\
\hline
\end{tabular}

Continued 
Table 1 Continued

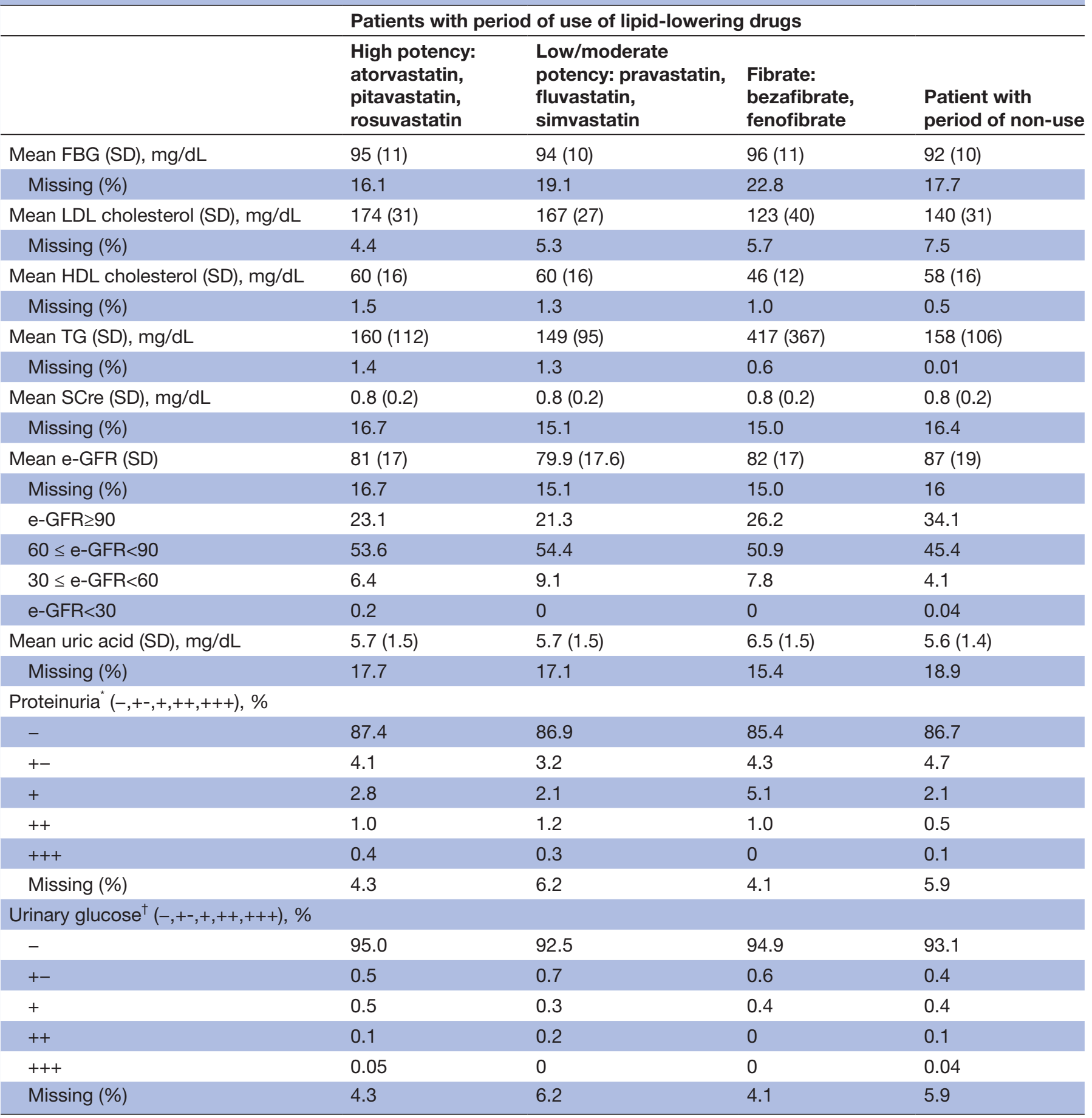

${ }^{*}$ The indices $(-,+-,+,++,+++)$ typically correspond to the qualitative values of $0,15,30,100$ and $250 \mathrm{mg} / \mathrm{dL}$ of proteinuria, respectively. †The indices $(-,+-,+,++,+++)$ typically correspond to $0,50,100,500$ and $2000 \mathrm{mg} / \mathrm{dL}$ of urinary glucose, respectively. BMI, body mass index; DBP, diastolic blood pressure; e-GFR, estimated glomerular filtration rate; HbA1c, haemoglobin A1c; FBG, fasting blood glucose; HDL, high-density lipoprotein; LDL, low-density lipoprotein; SBP, systolic blood pressure; SCre, serum creatinine; TG, triglyceride.

incidence rate of new-onset diabetes was estimated as 22.6 per 1000 person-years during the period of non-use in our study, the absolute increase of the rate potentially due to the use of statins, corresponding to HR of 1.5 to 3.1 , may be approximately 10 to 45 per 1000 personyears.
Previous studies on the association between the use of statins and new-onset diabetes have been inconclusive. In some of the observational cohort studies, ${ }^{525}$ and a meta-analysis of randomised placebo-controlled trials, ${ }^{4}$ the risk of new-onset diabetes was not different between low and high potency statins. On the other hand, in a 


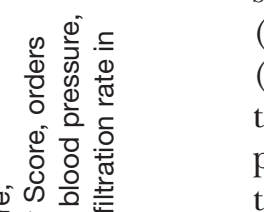

study using the pharmacy claims by Zaharan et al, the HR (1.3 to 1.4) of new-onset diabetes in high potency statins (atorvastatin and rosuvastatin) was slightly higher than the HR (1.0 to 1.1) in low potency statins (fluvastatin, pravastatin and simvastatin). ${ }^{26}$ Our findings suggest that the risk of new-onset diabetes in high potency statins may be higher than that of low potency statins, in line with the findings of Zaharan et al. ${ }^{26}$ These discrepancies may be explained by the different outcome definitions used among studies.

Our study did not find fibrates (fenofibrate and bezafibrate) to be associated with an increased risk of new-onset diabetes compared with that for non-use. However, we cannot rule out a possible increased risk due to small numbers of patients taking fibrates. There have been few studies on the risk of diabetes in patients prescribed with fibrates. In a randomised controlled trial on cardiovascular events and a claims-based study in Taiwan, ${ }^{89}$ fibrates were not associated with an increased risk of new-onset diabetes. However, the observational study by Flory et al indicated that the risk of new-onset diabetes due to bezafibrate use was different to that of other fibrates. ${ }^{27}$ In our findings, the risk of new-onset diabetes due to fibrates tended to be high. However, the number of patients with new-onset diabetes was small (19) and the HR had a wide CI. Therefore, it is quite possible that the higher risk of fibrates compared with non-use was obtained just by chance in the current study.

Both statins and fibrates are known to affect glucose homeostasis. ${ }^{28} 29$ The insulin-sensitising action of adiponectin results from a decrease in hepatic gluconeogenesis and an increase in muscle glucose transport. ${ }^{30}$ Hypoadiponectin may be associated with the incidence of diabetes. ${ }^{31}{ }^{32}$ In a meta-analysis comparing fibrates and statins for elevation of circulating adiponectin, a non-significant negative trend was indicated between changes in plasma adiponectin and glucose levels. ${ }^{33}$ Fibrates $^{34}$ and statins ${ }^{35} 36$ lowered the adiponectin level in some studies. However, another meta-analysis suggested that statin and fibrate therapy may increase circulating adiponectin. ${ }^{37} 38$

Our study has several limitations. First, this was not a randomised controlled trial but an observational cohort study and therefore we cannot exclude the possibility that the results are biased due to the residual confoundings although the potential confounding effects were adjusted in the analysis as much as possible. In particular, the information on some of known risk factors including smoking, alcohol consumption and physical exercise was not available and we could not adjust for them. Second, our study population included only those who are working in relatively large corporations and their dependents, aged 20 to 74 years. In addition, as the health screening was not compulsory for the employee's relatives, the proportion of the relatives who had the health screening data was small (approximately 30\%) and those who had the health screening may be systematically different from those who did not. For example, those who had the health screening may be more health orientated. Therefore, our findings 
Table 3 Association between individual lipid-lowering drugs and new-onset diabetes

HR (95\% Cl)

\begin{tabular}{llll}
\hline Drug class & Unadjusted & Adjusted for age and sex & Multivariate* adjusted \\
\hline Non-use & 1.00 & 1.00 & 1.00 \\
Pravastatin & $4.86(3.49$ to 6.75$)$ & $3.40(2.44$ to 4.74$)$ & $1.93(1.32$ to 2.82$)$ \\
\hline Fluvastatin & $4.81(2.11$ to 10.94$)$ & $3.31(1.44$ to 7.59$)$ & $2.25(1.04$ to 4.90$)$ \\
\hline Simvastatin & $3.56(1.52$ to 8.46$)$ & $2.37(0.99$ to 5.62$)$ & $1.53(0.64$ to 3.68$)$ \\
\hline Atorvastatin & $4.99(3.59$ to 6.93$)$ & $3.37(2.41$ to 4.71$)$ & $2.15(1.52$ to 3.04$)$ \\
Rosuvastatin & $5.56(4.30$ to 7.19$)$ & $4.00(3.09$ to 5.18$)$ & $2.70(1.99$ to 3.66$)$ \\
\hline Pitavastatin & $7.96(5.85$ to 10.82$)$ & $5.74(4.21$ to 7.84$)$ & $3.11(2.20$ to 4.40$)$ \\
Bezafibrate & $4.24(2.37$ to 7.59$)$ & $3.14(1.72$ to 5.71$)$ & $1.54(0.79$ to 3.00$)$ \\
\hline Fenofibrate & $4.53(2.25$ to 9.13$)$ & $3.45(1.71$ to 6.97$)$ & $1.82(0.82$ to 4.02$)$ \\
\hline
\end{tabular}

${ }^{*}$ Adjusted for age, sex, co-medications (nicotinic acid, antihypertensive drugs, antipsychotic drugs and steroid), concurrent diseases (myocardial infarction, chronic heart failure, cerebrovascular disease, renal disease, liver disease, pulmonary disease, hypertension, polycystic ovarian syndrome and cancer), metabolic syndrome, Charlson Comorbidity Score, orders (haemoglobin A1c, triglyceride, total cholesterol, low-density lipoprotein (LDL) cholesterol and high-density lipoprotein (HDL) cholesterol) in claims data and body mass index, blood pressure, fasting blood glucose, haemoglobin A1c, LDL cholesterol, HDL cholesterol, triglyceride, serum creatinine, uric acid, urinary protein and urinary glucose, estimated glomerular filtration rate in data in health screening.

might not be generalisable to those working in small corporations and self-employed, of older ages, and those who did not have the health screening. Third, the diagnosis code for diabetes was not validated in our database. However, the results of our sensitivity analyses, where the outcome was defined using an antidiabetic drug only, were similar to those in the primary analysis where the outcome was defined by a diagnosis code of diabetes or the use of an antidiabetic drug. In general, the outcome defined by the initiation of a drug may be better than the outcome defined by the diagnosis code. ${ }^{39} 40$ Fourth, the mean follow-up period (approximately 4 to 5 months) of LLDs in this study was relatively short, and we could not determine the risks associated with the longer use from the current study. A comparison with the previous studies with a longer follow-up period may also be difficult.

\section{Low potency statin Hazard ratio $(95 \% \mathrm{CI})$ \\ High potency statin Hazard ratio $(95 \% \mathrm{CI})$ \\ Fibrate \\ Hazard ratio $(95 \% \mathrm{CI})$}

\section{Outcome defined by diagnosis and/or drug}

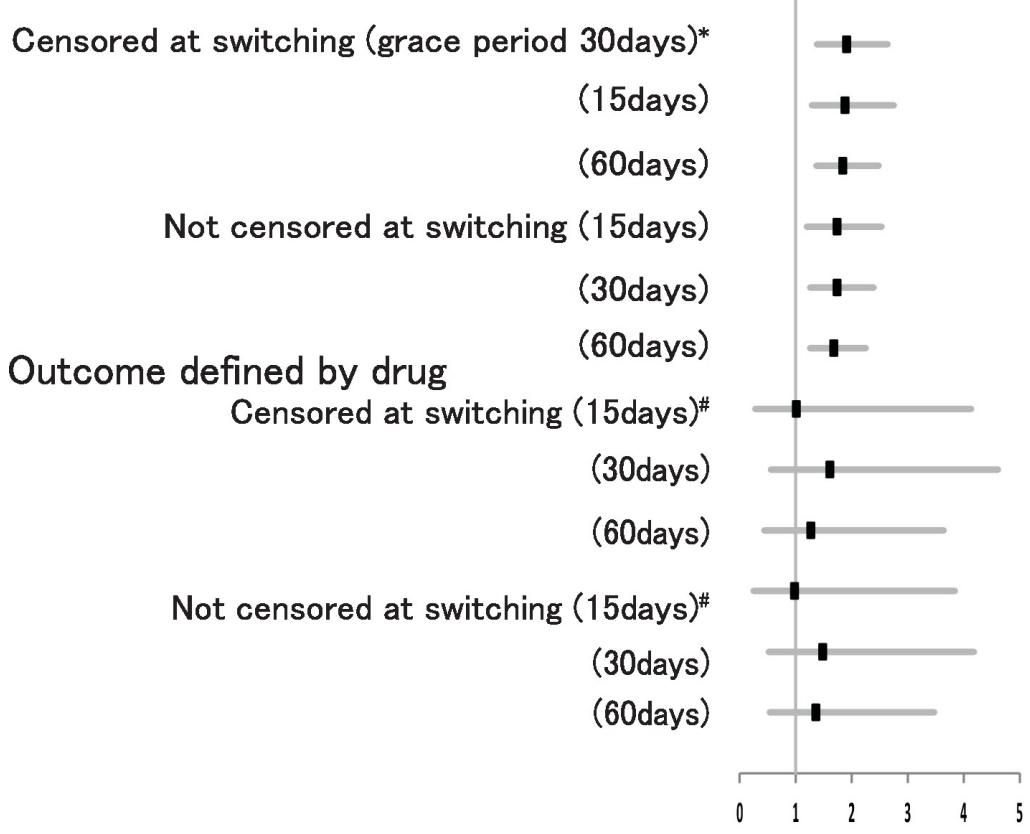

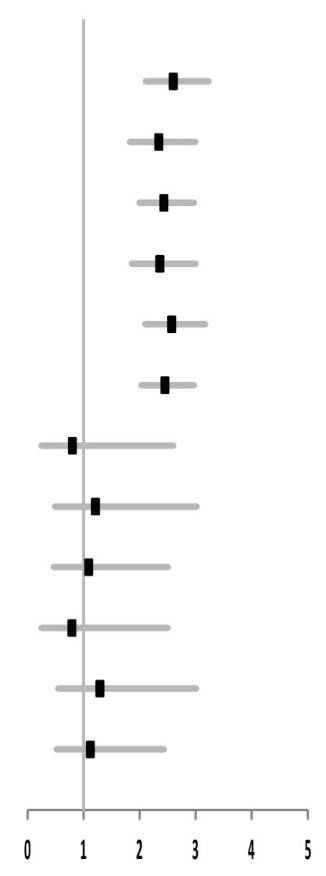

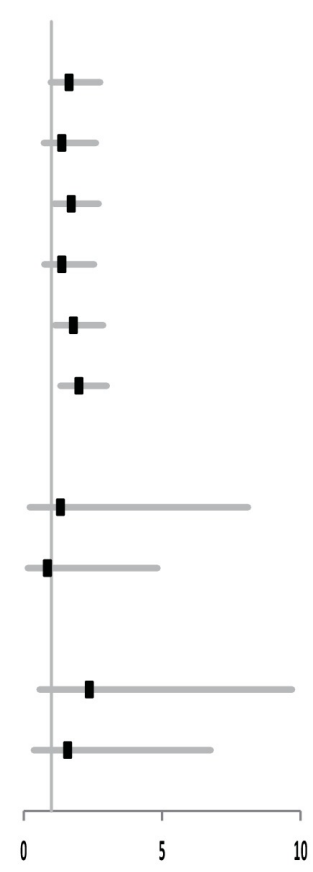

Figure 2 Sensitivity analysis.*: primary analysis\#: for the grace period of 15 days, no case was observed in those with fibrates. Outcome defined by diagnosis and/or drug; outcome defined by a diagnosis code of diabetes and/or new use of an antidiabetic drug; outcome defined by drug; outcome defined by new use of an antidiabetic drug disregarding the diagnosis code. 
However, in the population-based study by Carter $e t a l{ }^{6}$ where the risk of new-onset diabetes was found to be increased among patients treated with statins, the median follow-up period was also short ( $<1$ year). There were case reports where the patient had new-onset diabetes in 1 to 2 months after the administration of a statin. ${ }^{41}$ Fifth, the relatively high frequency of physician visits during the period of drug treatment as compared with that during the period of non-use might have introduced a detection bias. However, some of our findings such as the difference in the magnitude of the risk of new-onset diabetes between high and low potency statins could not be accounted for by the detection bias. Sixth, we could not examine the dose-response relationships, as the number of patients who had new-onset diabetes was not large enough to examine the details, such as any risk between the use of individual statins and dose-response relationships.

Our study has several strengths. We could adjust for HbA1c and FBG, essential in the diagnosis of diabetes, as well as for BMI, blood pressure, UA, LDL cholesterol, HDL cholesterol, TG, proteinuria and urinary glucose measured on the index date and on the date of new use of an LLD. ${ }^{7}$ These variables have been unavailable in most previous studies using claims data. Statins were associated with an increased risk of new-onset diabetes, even after being adjusted for all available confounders, including covariates, although there may be residual confounders as information was not available on some potential confounders such as smoking, alcohol consumption and physical exercise.

In conclusion, we found that the use of LLDs (low and high potency statins and potentially fibrates as well) in a Japanese workers and their dependents aged 20 to 74 was associated with new-onset diabetes, compared with non-use. The risk of new-onset diabetes in high potency statins was higher than that in low potency statins. Further studies are needed to understand the association between new-onset diabetes and fibrates. More studies are needed to evaluate the association between new-onset diabetes and the use of LLDs in the elderly, and in association with individual LLDs.

Contributors All authors contributed to the conception and design of the study. NO, TS and KK contributed to the acquisition of the data. NO, SS and KK contributed to the analysis and the interpretation of the data. NO, SS and KK drafted the manuscript. NO and KK are the guarantors.

Funding This research received no specific grant from any funding agency in the public, commercial or not-for-profit sectors.

\section{Competing interests None declared.}

Ethics approval This study was approved by the Ethical Committee of Nihon University School of Pharmacy (No. 14011)

Provenance and peer review Not commissioned; externally peer reviewed.

Data sharing statement No additional data are available.

Open Access This is an Open Access article distributed in accordance with the Creative Commons Attribution Non Commercial (CC BY-NC 4.0) license, which permits others to distribute, remix, adapt, build upon this work non-commercially, and license their derivative works on different terms, provided the original work is properly cited and the use is non-commercial. See: http://creativecommons.org/ licenses/by-nc/4.0/ (c) Article author(s) (or their employer(s) unless otherwise stated in the text of the article) 2017. All rights reserved. No commercial use is permitted unless otherwise expressly granted.

\section{REFERENCES}

1. National Health survey in 2011. 2011. (In Japanese) http://www. mhlw.go.jp/toukei/saikin/hw/kanja/11/dl/04.pdf.

2. Stone NJ, Robinson JG, Lichtenstein AH, et al. 2013 ACC/AHA Cholesterol Guideline Panel. Treatment of blood cholesterol to reduce atherosclerotic cardiovascular disease risk in adults: synopsis of the 2013 American College of Cardiology/American Heart Association cholesterol guideline. Ann Intern Med 2014;160:339-43.

3. Hu M, Cheung BM, Tomlinson B. Safety of statins: an update. Ther Adv Drug Saf 2012;3:133-44.

4. Sattar N, Preiss D, Murray HM, et al. Statins and risk of incident diabetes: a collaborative meta-analysis of randomised statin trials. Lancet 2010;375:735-42.

5. Culver AL, Ockene IS, Balasubramanian R, et al. Statin use and risk of diabetes mellitus in postmenopausal women in the Women's Health Initiative. Arch Intern Med 2012;172:144-52.

6. Carter AA, Gomes T, Camacho X, et al. Risk of incident diabetes among patients treated with statins: population based study. BMJ 2013;346:f2610.

7. American Diabetes Association. Standards of medical care in diabetes--2011. Diabetes Care 2011;34 Suppl 1:S11-S61.

8. Keech A, Simes RJ, Barter P, et al. Effects of long-term fenofibrate therapy on cardiovascular events in 9795 people with type 2 diabetes mellitus (the FIELD study): randomised controlled trial. Lancet 2005;366:1849-61.

9. Lee $\mathrm{CY}$, Huang $\mathrm{KH}$, Lin $\mathrm{CC}$, et al. A neutral risk on the development of new-onset diabetes mellitus (NODM) in Taiwanese patients with dyslipidaemia treated with fibrates. ScientificWorldJournal 2012;2012:1-5.

10. Chen CW, Chen TC, Huang KY, et al. Differential impact of statin on new-onset diabetes in different age groups: a population-based case-control study in women from an asian country. PLoS One 2013;8:e71817.

11. Rha SW, Choi BG, Seo HS, et al. Impact of statin use on development of New-Onset Diabetes Mellitus in Asian Population. Am J Cardiol 2016;117:382-7.

12. Ishikawa KB. Medical Big Data for Research Use: current status and related issues. Japan Med Assoc J 2016;59:110-24.

13. Kimura S, Sato T, Ikeda S, et al. Development of a database of health insurance claims: standardization of disease classifications and anonymous record linkage. J Epidemiol 2010;20:413-9.

14. National drug code. http://apps.who.int/medicinedocs/en/d/ Js19548ja/

15. Suka M, Yoshida K, Matsuda S. Effect of annual health checkups on medical expenditures in japanese middle-aged workers. J Occup Environ Med 2009;51:456-61.

16. Hata $\mathrm{Y}$, Mabuchi H, Saito $\mathrm{Y}$, et al. Working Committee on JAS Guideline for Diagnosis and Treatment of Hyperlipidemias. Report of the Japan Atherosclerosis Society (JAS) Guideline for diagnosis and treatment of Hyperlipidemia in Japanese adults. J Atheroscler Thromb 2002;9:1-27.

17. Teramoto T, Sasaki J, Ueshima H, et al. Japan Atherosclerosis Society (JAS) Committee for Epidemiology and Clinical Management of Atherosclerosis. Diagnostic criteria for dyslipidemia. Executive summary of Japan Atherosclerosis Society (JAS) guideline for diagnosis and prevention of atherosclerotic cardiovascular diseases for Japanese. J Atheroscler Thromb 2007;14:155-8.

18. Law MR, Wald NJ, Rudnicka AR. Quantifying effect of statins on low density lipoprotein cholesterol, ischaemic heart disease, and stroke: systematic review and meta-analysis. BMJ 2003;326:1423-30.

19. Chung $\mathrm{YH}$, Lee $\mathrm{YC}$, Chang $\mathrm{CH}$, et al. Statins of high versus low cholesterol-lowering efficacy and the development of severe renal failure. Pharmacoepidemiol Drug Saf 2013;22:583-92.

20. Christensen S, Johansen MB, Christiansen CF, et al. Comparison of Charlson comorbidity index with SAPS and APACHE scores for prediction of mortality following intensive care. Clin Epidemiol 2011;3:203-11.

21. Matsuo S, Imai E, Horio M, et al. Revised equations for estimated GFR from serum creatinine in Japan. Am J Kidney Dis 2009;53:982-92.

22. Sicras A, Rejas J, Navarro R, et al. Metabolic syndrome in bipolar disorder: a cross-sectional assessment of a Health Management Organization database. Bipolar Disord 2008;10:607-16. 
23. Robins JM, Hernán MA, Brumback B. Marginal structural models and causal inference in epidemiology. Epidemiology 2000;11:550-60.

24. Danaei G, Rodríguez LA, Cantero OF, et al. Observational data for comparative effectiveness research: an emulation of randomised trials of statins and primary prevention of coronary heart disease. Stat Methods Med Res 2013;22:70-96.

25. Ma T, Tien L, Fang CL, et al. Statins and new-onset diabetes: a retrospective longitudinal cohort study. Clin Ther 2012;34:1977-83.

26. Zaharan NL, Williams D, Bennett K. Statins and risk of treated incident diabetes in a primary care population. Br J Clin Pharmacol 2013;75:1118-24.

27. Flory JH, Ellenberg S, Szapary PO, et al. Antidiabetic action of bezafibrate in a large observational database. Diabetes Care 2009;32:547-51.

28. Kostapanos MS, Liamis GL, Milionis HJ, et al. Do statins beneficially or adversely affect glucose homeostasis? Curr Vasc Pharmacol 2010;8:612-31.

29. Okopień B, Krysiak R, Herman ZS. Effects of short-term fenofibrate treatment on circulating markers of inflammation and hemostasis in patients with impaired glucose tolerance. J Clin Endocrinol Metab 2006;91:1770-8.

30. Xita N, Tsatsoulis A. Adiponectin in diabetes mellitus. Curr Med Chem 2012:19:5451-8.

31. Kadowaki T, Yamauchi T, Kubota N, et al. Adiponectin and adiponectin receptors in insulin resistance, diabetes, and the metabolic syndrome. J Clin Invest 2006;116:1784-92.

32. Wannamethee SG, Lowe GD, Rumley A, et al. Adipokines and risk of type 2 diabetes in older men. Diabetes Care 2007;30:1200-5.

33. Sahebkar A. Head-to-head comparison of fibrates versus statins for elevation of circulating adiponectin concentrations: a systematic review and meta-analysis. Metabolism 2013;62:1876-85.
34. Ryan KE, McCance DR, Powell L, et al. Fenofibrate and pioglitazone improve endothelial function and reduce arterial stiffness in obese glucose tolerant men. Atherosclerosis 2007;194:e123-e130.

35. Koh KK, Oh PC, Sakuma I, et al. Rosuvastatin dose-dependently improves flow-mediated dilation, but reduces adiponectin levels and insulin sensitivity in hypercholesterolemic patients. Int $\mathrm{J}$ Cardiol 2016;223:488-93.

36. Thongtang $\mathrm{N}, \mathrm{Ai} \mathrm{M}$, Otokozawa $\mathrm{S}$, et al. Effects of maximal atorvastatin and rosuvastatin treatment on markers of glucose homeostasis and inflammation. Am J Cardiol 2011;107:387-92.

37. Chruściel P, Sahebkar A, Rembek-Wieliczko M, et al. Impact of statin therapy on plasma adiponectin concentrations: a systematic review and meta-analysis of 43 randomized controlled trial arms. Atherosclerosis 2016;253:194-208.

38. Sahebkar A, Watts GF. Fibrate therapy and circulating adiponectin concentrations: a systematic review and metaanalysis of randomized placebo-controlled trials. Atherosclerosis 2013;230:110-20.

39. Park BJ, Stergachis A. Automated Databases in Pharmacoepidemiologic Studies. In: Hartzema AG, Tilson HH, Chan KA, eds. Pharmacoepidemiology and therapeutic risk management : Harvey Whitney Books, 2008.

40. Strom BL. How Should One Perform Pharmacoepidemiologic Studies? Choosing Among the Available Alternatives. In: Strom BL, Kimmel SE, Hennessy S, eds. Textbook of Pharmacoepidemiology: Willey Blackwell, 2013

41. Sasaki J, Iwashita M, Kono S. Statins: beneficial or adverse for glucose metabolism. J Atheroscler Thromb 2006;13:123-9. 